\title{
Multimodal vibration damping using energy transfer
}

\author{
A. Cherif ${ }^{1} \cdot$ M. Meddad ${ }^{1}$ - A. Eddiai ${ }^{2}$ - A. Zouhair $^{2}$ • \\ A. Zawadzka ${ }^{3}$ A. Migalska-Zalas ${ }^{4}$
}

Received: 15 November 2015/Accepted: 4 February 2016/Published online: 16 April 2016

(C) Springer Science+Business Media New York 2016

\begin{abstract}
The vibration control using the piezoelectric elements is an area interesting for many industrial sectors. Within this framework, we propose an improved control technique based in synchronized switch damping by energy transfer. It realizes the energy transfer using storage capacitances and switches synchronized with the structure modal coordinates or piezo-voltages. These switches produce either a voltage inversion on the piezoelements for damping or energy extraction purposes, or oscillating discharges between the piezoelements and the storage capacitances for energy transfer. This new method has an improvement in the modal damping technology SSDI-Max. Their performance is simulated with a model representative of a clamped plate with four piezoelectric elements coupled with the structural modes while taking into account realistic transfer losses. The damping effect is simulated in multi-modal with pulse or multi-sine excitation.
\end{abstract}

Keywords Vibration damping $\cdot$ Piezoelectric $\cdot$ Multimodal control $\cdot$ SSDI-Max $\cdot$ Energy transfer

This article is part of the Topical Collection on Advanced Materials for photonics and electronics.

Guest Edited by Bouchta Sahraoui, Yahia Boughaleb, Kariem Arof, Anna Zawadzka.

\section{A. Migalska-Zalas}

aniazal@op.pl

1 Département d'électromécanique Université Bachir el Ibrahimi BBA, Laboratoire LAS, Université Ferhat Abbas, 19000 Sétif, Algeria

2 Laboratoire de Physique de la Matière Condensée, Faculté des Sciences Ben M Sik, Université Hassan II, Casablanca, Morocco

3 Institute of Physics, N. Copernicus University, Grudziądzka 5, 87-100 Toruń, Poland

4 Institute of Physics, Faculty of Mathematics and Natural Sciences, J. Dlugosz Academy of Czestochowa, Al. Armii Krajowej 13/15, Czestochowa, Poland 


\section{Introduction}

Undesired mechanical vibration harms the running mechanical equipment's. It could lead to material fatigue, deterioration of system performance, also increase the noise level. Particularly, the structure could be damaged due to the high amplitude vibration when the excitation just locates around structure eigenvalue frequencies. Several catastrophes such as bridge collapse, airplane crash have been ascribed to vibration caused problems. Thus, vibration control becomes to an urgent issue for the mechanical engineers. In the past decades, such techniques grew rapidly in kinds of industry domain. These approaches can be generally classified as three catalogs, which are passive control, active control and semiactive or semi-passive control.

Passive control is the earliest developed vibration control methods. These methods are easy to implement, sensor and power needless and unconditional stable. The main disadvantage of the passive control is that the control bandwidth is too narrow. Once the structure dynamic properties changes, the control system would need to be returned. In addition, the passive control need to add non negligible mass to the structure which could be unacceptable in aerospace field. Active control is developed based on the development of the computer science. The control system need sensors to monitor the displacement or velocity of the structure. The sensed signals then send to a controller in order to obtain a control signal by employing specific algorithms. The control signal would be amplified by power amplifier or directly exert on actuators to generate feedback force on the structure which usually has the opposite phase with the external excitation. Among kinds of semiactive and semi-passive vibration control methods, synchronized switch damping (SSD) techniques are proved to be an effective treatment. Compared with the passive methods, the system as the immunity against the structure dynamic properties shift due to the environmental change. They are also compact, lightweight which is convenient to apply to specific structure with weight or size restriction. Compared with the active control, SSD control system is very simple to implement and can easily be self-powered from the vibration itself. In these techniques, the switch in the circuit is intermittently switched leading to a non-linear voltage processing (Richard et al. 1999; Ji et al. 2014; Lefeuvre et al. 2006; Badel et al. 2006; Eddiai et al. 2012). The piezo-force induced by such voltage always shows an opposite sign with the structure velocity which leading the vibration suppression on the structure. Such behavior is similar with the well-known direct velocity feedback control.

The usual SSD technique performs well in mono-modal excitation; however, it hardly deals with the cases of the multimodal and complex vibrations. The limitation is due to the difficulty of defining the proper switching time in order to have optimal voltage magnification as well as proper phase according to the structure motion. To overcome this limitation, an alternative technique named Modal-SSDI was developed by Harari et al. (2009a, b). It consists of synchronizing the switch sequence on a given modal coordinate instead of the voltage. It therefore combines the simplicity, the robustness and low power operation of SSDI techniques, with the possibility of mode targeting and precision of active control strategies. Modal-SSDI takes advantage of a modal model of the structure to design a modal observer permitting the calculation of the modal coordinates characterizing the motional state of the structure. Based on the Modal-SSDI, SSDI-Max method (Chérif et al. 2012, 2015, 2013) was developed to deal with a multimodal control problem and to improve the damping performance by improving the piezoelectric element voltage amplitude. The principle of this method is developed with the objective of maximizing the 
self-generated voltage amplitude. Each time the chosen modal coordinate reaches an extremum, the device waits within a given limited time window for the next voltage extremum or for a significant voltage increase before to trigger the switch sequence. In this method, the extra voltage is gathered on the various non-controlled modes of the structure by the piezoelectric element itself.

In this article, based on SSDI-Max technique, a new global approach for improved vibration damping of smart structure, based on global energy redistribution by means of a network of piezoelectric elements. The objective of this work is to propose a new approach to increase the piezoelectric voltage in order to improve the damping performance. In this approach, the extra energy used to improve this voltage is gathered on the various modes of the structure using an interconnected piezoelectric element network. The network topologies developed in this paper is named SST-Max for "synchronized switch damping by energy transfer using SSDI-Max". Performance evaluations and comparisons are performed on a model representative of a clamped plate equipped with piezoelectric elements in the case of multimodal motion. Compared to the SST developed by Wu et al. (2013), simulation results and a global theoretical model are proposed demonstrating the relationship between the achievable damping improvement and the ratio of transferred energy to the structure mechanical energy.

\section{The semi-active Modal-SSDI-Max technique}

Damping performance is strongly dependent on the piezoelectric voltage amplitude. The Modal-SSDI-Max was developed (Chérif et al. 2012, 2015) with the objective of maximizing the self-generated voltage amplitude by a proper definition of the switch instants according to the chosen modal coordinate extremum. Compared with Modal-SSDI control method, the strategy of Modal-SSDI-Max consists in delaying the switch instant to the next voltage extremum immediately following the targeted modal coordinate extremum. It means that Modal-SSDI-Max also relies on a modal model and modal observer permitting the calculation of the modal coordinates to synchronize the switch with the targeted mode. In order to avoid a desynchronization with the target modal displacement, a time window is set up to limit the delay. Figure 1 illustrates the strategy of this technique in the various

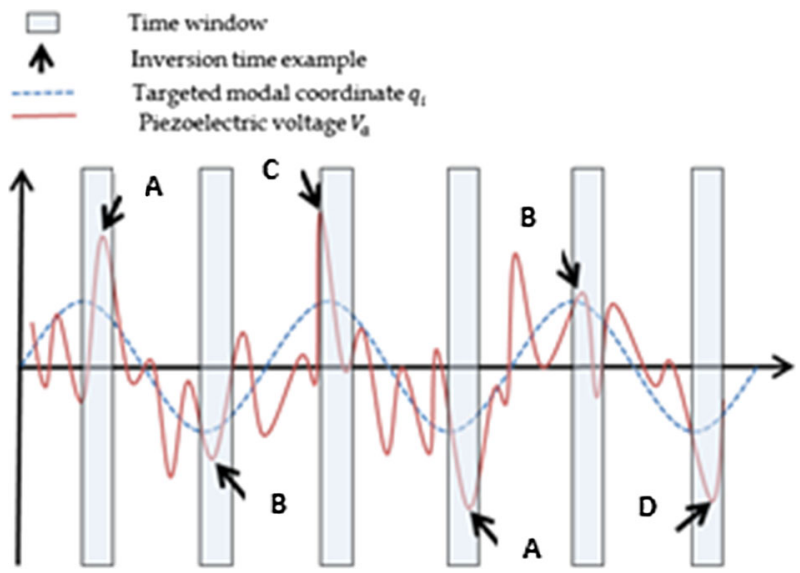

Fig. 1 Strategy of Modal-SSDI-Max vibration control (Chérif et al. 2012) 
cases. The grey are a materializes the time window which is initiated for each extremum of the targeted modal coordinate. Points A, B, C and D illustrate the example of chosen switching time, which is indicated by the arrow.

Point A It is necessary to wait for the next positive maximum of the voltage to ensure the optimal voltage amplitude. Immediately switching would result in a wrong phase for the voltage of the piezoelectric actuator.

Point $B$ Even if the voltage has the right sign, it is better to wait for the next voltage extremum to obtain a higher amplitude of the voltage.

Point $C$ Voltage is decreasing at the beginning of the window, so it's better to switch immediately.

Point $D$ If no voltage extremum with the correct sign is located in the time window, the switch should be closed at the end of the time window, thus limiting the resulting voltage phase shift.

\section{Smart structure definition}

\subsection{Smart structure modeling}

The electromechanical behavior equations of a smart structure using usual assumption are as follows (Harari et al. 2009a, b):

$$
\begin{gathered}
m \ddot{\delta}+c \dot{\delta}+k^{E} \delta=-\alpha V+\beta F \\
I=\alpha^{t} \dot{\delta}-C_{0} \dot{V}
\end{gathered}
$$

with $\delta$ the nodal displacement vector, $m, c$, and $k^{E}$ are the mass, damping, and stiffness matrices, respectively, when the piezoelectric patches are in short circuit, $\alpha$ is the electromechanical coupling matrix, $V$ is voltage vector of the $i$ piezoelectric patches, $I$ is the electric current vector, and $C_{0}$ is the diagonal capacitance matrix. $F$ is the force applied to the system.

Using the following variable change where $\phi$ is the mode shape matrix limited to $n$ modes and $q$ the modal displacement vector:

$$
\delta=\phi q
$$

The Eqs. (1) and (2) can be well represented by the projection in the modal basis by

$$
\begin{gathered}
m \ddot{q}+c \dot{q}+k^{E} q=-\theta V+\beta F \\
I=\theta^{t} \dot{q}-C_{0} \dot{V}
\end{gathered}
$$

where $\theta$ is the modal electromechanical coupling matrix with a $[n, i]$ matrix size. $\Theta$ is defined as follows

$$
\theta=\phi^{t} \alpha
$$

where $M, C$, and $K^{E}$ are the mass, damping, and stiffness modal matrices, respectively. The structure is assumed to be lightly damped, with proportional damping and decoupled modes. Equation (4) is normalized in order to have the modal mass matrix equal to identity. The modal matrices can therefore be written as a function of $\xi$ the modal damping vector, $\omega^{E}$ the short-circuit frequency vector, and $\omega^{D}$ the open circuit frequency vector as follows 


$$
M=I_{d} ; \quad C=2 \operatorname{diag}(\xi) \operatorname{diag}\left(\omega^{D}\right) ; \quad K^{E}=\operatorname{diag}\left(\left(\omega^{E}\right)^{2}\right)
$$

The multi-modal model of the structure can be therefore expressed in matrix form as follows:

$$
\begin{aligned}
& {\left[\begin{array}{ccc}
m_{1} & 0 & 0 \\
0 & \ddots & 0 \\
0 & 0 & m_{n}
\end{array}\right]\left[\begin{array}{c}
\ddot{q}_{1} \\
\vdots \\
\ddot{q}_{n}
\end{array}\right]+\left[\begin{array}{ccc}
c_{1} & 0 & 0 \\
0 & \ddots & 0 \\
0 & 0 & c_{n}
\end{array}\right]\left[\begin{array}{c}
\dot{q}_{1} \\
\vdots \\
\dot{q}_{2}
\end{array}\right]+\left[\begin{array}{ccc}
k_{1}^{E} & 0 & 0 \\
0 & \ddots & 0 \\
0 & 0 & k_{n}^{E}
\end{array}\right]\left[\begin{array}{c}
q_{1} \\
\vdots \\
q_{n}
\end{array}\right] } \\
= & {\left[\begin{array}{ccc}
\theta_{11} & \ldots & \theta_{1 z} \\
\vdots & \ddots & \vdots \\
\theta_{n 1} & \ldots & \theta_{n z}
\end{array}\right]\left[\begin{array}{c}
V_{1} \\
\vdots \\
V_{z}
\end{array}\right]+\left[\begin{array}{c}
\beta_{1} \\
\vdots \\
\beta_{n}
\end{array}\right] F } \\
& {\left[\begin{array}{c}
I_{1} \\
\vdots \\
I_{z}
\end{array}\right]=-\left[\begin{array}{ccc}
\theta_{11} & \ldots & \theta_{n 1} \\
\vdots & \ddots & \vdots \\
\theta_{1 z} & \ldots & \theta_{n z}
\end{array}\right]\left[\begin{array}{c}
\dot{q}_{1} \\
\vdots \\
\dot{q}_{n}
\end{array}\right]-\left[\begin{array}{ccc}
C_{01} & 0 & 0 \\
0 & \ddots & 0 \\
0 & 0 & C_{0 z}
\end{array}\right]\left[\begin{array}{c}
\dot{V}_{1} \\
\vdots \\
\dot{V}_{z}
\end{array}\right] }
\end{aligned}
$$

For the sake of simplicity and in order to use powerful numerical tools, the smart structure is modeled by Eqs. (10) and (11), which are still presented by using a state space formulated equation (Eq. 12), respectively.

$$
[P P]_{2 n \times 2 n}\left[\begin{array}{c}
\dot{q}_{1} \\
\vdots \\
\dot{q}_{n} \\
\ddot{q}_{1} \\
\vdots \\
\ddot{q}_{n}
\end{array}\right]_{2 n \times 1}=-[Q Q]_{2 n \times 2 n}\left[\begin{array}{c}
q_{1} \\
\vdots \\
q_{n} \\
\dot{q}_{1} \\
\vdots \\
\dot{q}_{n}
\end{array}\right]_{2 n \times 1}+[R R]_{2 n \times 2 n}\left[\begin{array}{c}
F \\
V_{1} \\
\vdots \\
V_{z}
\end{array}\right]_{(z+1) \times 1}
$$

Here,

$$
\begin{aligned}
{[P P]_{2 n \times 2 n}=} & {\left[\begin{array}{ccc}
{\left[\begin{array}{ccc}
0 & \ldots & 0 \\
\vdots & \ddots & \vdots \\
0 & \ldots & 0
\end{array}\right]_{n \times n}} & {\left[\begin{array}{ccc}
m_{1} & 0 & 0 \\
0 & \ddots & \vdots \\
0 & 0 & m_{n}
\end{array}\right]_{n \times n}} \\
{\left[\begin{array}{ccc}
1 & 0 & 0 \\
0 & \ddots & 0 \\
0 & 0 & 1
\end{array}\right]_{n \times n}} & {\left[\begin{array}{ccc}
0 & \ldots & 0 \\
\vdots & \ddots & \vdots \\
0 & \ldots & 0
\end{array}\right]_{n \times n}}
\end{array}\right] } \\
{[Q Q]_{2 n \times 2 n}=} & {\left[\begin{array}{ccc}
{\left[\begin{array}{ccc}
k_{1}^{E} & \ldots & 0 \\
\vdots & \ddots & \vdots \\
0 & \ldots & k_{n}^{E}
\end{array}\right]_{n \times n}} & {\left[\begin{array}{ccc}
c_{1} & 0 & 0 \\
0 & \ddots & \vdots \\
0 & 0 & c_{n}
\end{array}\right]_{n \times n}} \\
{\left[\begin{array}{ccc}
0 & \ldots & 0 \\
\vdots & \ddots & \vdots \\
0 & \ldots & 0
\end{array}\right]_{n \times n}} & {\left[\begin{array}{ccc}
1 & 0 & 0 \\
0 & \ddots & 0 \\
0 & 0 & 1
\end{array}\right]_{n \times n}}
\end{array}\right] }
\end{aligned}
$$




$$
\begin{gathered}
{[R R]_{2 z \times(z+1)}=\left[\begin{array}{c}
{\left[\begin{array}{c}
1 \\
\vdots \\
1
\end{array}\right]_{n \times 1}\left[\begin{array}{ccc}
\theta_{11} & \ldots & \theta_{1 z} \\
\vdots & \ddots & \vdots \\
\theta_{n 1} & \ldots & \theta_{n z}
\end{array}\right]_{n \times z}} \\
{\left[\begin{array}{c}
0 \\
\vdots \\
0
\end{array}\right]_{n \times 1}\left[\begin{array}{ccc}
0 & \ldots & 0 \\
\vdots & \ddots & \vdots \\
0 & \ldots & 0
\end{array}\right]_{n \times z}}
\end{array}\right]} \\
{[R]^{-1}\left[\begin{array}{c}
V_{1} \\
\vdots \\
V_{z}
\end{array}\right]_{z \times 1}=-[\theta]_{z \times n}^{T}\left[\begin{array}{c}
\dot{q}_{1} \\
\vdots \\
\dot{q}_{n}
\end{array}\right]_{n \times 1}-\left[C_{0}\right]_{z \times z}\left[\begin{array}{c}
\dot{V}_{1} \\
\vdots \\
\dot{V}_{z}
\end{array}\right]_{z \times 1}}
\end{gathered}
$$

Here,

$$
\begin{aligned}
{[R]_{z \times z}^{-1}=} & {\left[\begin{array}{ccc}
R_{1} & 0 & 0 \\
0 & \ddots & 0 \\
0 & 0 & R_{z}
\end{array}\right]_{z \times z}^{-1} ; \quad[\theta]_{z \times n}^{T}=\left[\begin{array}{ccc}
\theta_{11} & \ldots & \theta_{n 1} \\
\vdots & \ddots & \vdots \\
\theta_{1 z} & \ldots & \theta_{n z}
\end{array}\right]_{z \times n} ; } \\
{\left[C_{0}\right]_{z \times z} } & =\left[\begin{array}{ccc}
C_{01} & 0 & 0 \\
0 & \ddots & 0 \\
0 & 0 & C_{0 z}
\end{array}\right]_{z \times z}
\end{aligned}
$$

Considering mechanical and electrical equations, the state vector $x(t)$, control vector $u(t)$ and the output vector $y(t)$, which also correspond to the special state matrices term $A$, $B, C$ and $D$ :

$$
\begin{aligned}
& \dot{x}(t)=A x(t)+B u(t) \\
& y(t)=C x(t)+D u(t)
\end{aligned}
$$

\subsection{Smart structure definition}

Figure 2 illustrates the type of structure that is considered in the development of this work. It is a steel plate clamped on the four edges on a metallic frame. Four PZT patches (P189 hard PZT type) are attached on the lateral edges along the clamping frame. The four piezoelectric elements are globally coupled with the various mode of the structure. This structure has been fully characterized using usual techniques: resonance frequencies for the various open-circuited and short-circuited conditions for all the modes as well as voltage to modal displacement transfer function evaluation for all the piezoelements and all the modes. The dimensions, material properties of the steel plate and piezoelectric patches are gathered in Table 1. Details of the plate construction and characterization are given in Guyomar et al. (2008). Among the many low frequency modes that have been experimentally observed, only the four principal modes have been considered in the modal model, therefore $n=4$. These modes are considered as principal in the sense that they do present the stronger electromechanical couplings. As a consequence, when the structure is excited, the voltage is a linear combination of the four considered 


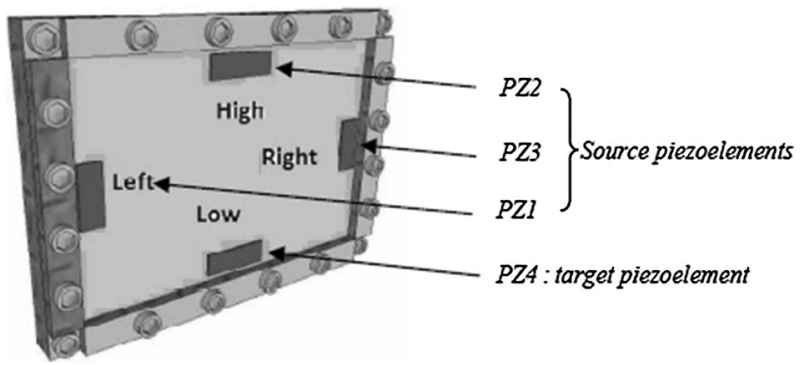

Fig. 2 The clamped steel plate with 4 with piezoelectric elements bonded at the lateral clamping edges

Table 1 The material properties of the steel plate and piezoelectric patch

\begin{tabular}{lll}
\hline Steel plate & Length & $0.6 \mathrm{~m}$ \\
& Width & $0.4 \mathrm{~m}$ \\
& Thickness & $1 \mathrm{~mm}$ \\
& Young modules & $210 \mathrm{GPa}$ \\
& Density & $7500 \mathrm{~kg} / \mathrm{m}^{3}$ \\
Piezoelectric elements & Poisson ratio & 0.345 \\
& Length & $0.12 \mathrm{~m}$ \\
& Width & $0.04 \mathrm{~m}$ \\
& Thickness & $0.6 \mathrm{~mm}$ \\
& Density & $7650 \mathrm{~kg} / \mathrm{m}^{3}$ \\
& Elastic compliance constant & $S_{11}^{E}=10.66 \mathrm{~Pa}$ \\
& Permittivity & $T$ \\
& Piezoelectric coefficient & $\varepsilon_{33}^{-1}=10.17 \mathrm{nF} / \mathrm{m}$ \\
& & $d_{31}=-108 \mathrm{pC} / \mathrm{N}$ \\
\hline
\end{tabular}

Table 2 Resonance frequencies and mechanical quality factor of the four considered modes of the smart structure (a) and piezoelectric elements blocked capacitances (b)

\begin{tabular}{lcc}
\hline Mode & $Q_{\mathrm{m}}$ & Frequency (Hz) \\
\hline (a) & & \\
1 & 90 & 96 \\
2 & 90 & 175 \\
3 & 180 & 204 \\
4 & 250 & 228 \\
\hline Piezoelement & & Blocked capacitance $C_{0}(\mathrm{nF})$ \\
\hline (b) & & \\
PZ1 & & \\
PZ2 & & \\
PZ3 & 71 & \\
PZ14 & 71 & \\
\hline
\end{tabular}

frequencies, such as in the considered model. Table 2 lists the four considered mode frequencies and mechanical quality factors and piezoelectric element blocked capacitances. 


\section{Energy transfer conception}

\subsection{Operative energy}

The energy balance equation within the structure. It also can be given as in Eq. (13) for a given modal coordinate.

$$
\left[\frac{1}{2} m \dot{q}^{2}\right]_{0}^{T}+\left[\frac{1}{2} k^{E} q^{2}\right]_{0}^{T}+\int_{0}^{T} C \dot{q}^{2} d t-\int_{0}^{T} \theta V \dot{q} d t=\int_{0}^{T} F \dot{q} d t
$$

The $\int_{0}^{T} \theta V \dot{q} d t$ is also considered as the switching damping energy or extracted energy which is composed of electrostatic energy stored on the piezoelectric element capacitance and the energy absorbed by the electric circuit which is connected with the piezoelectric elements as shown in Eq. (14).

$$
\int_{0}^{T} \theta V \dot{q} d t=\left[\frac{1}{2} C_{0} V^{2}\right]_{0}^{T}+\int_{0}^{T} V I d t
$$

It is remarkable that the electrostatic energy, so-called operative energy is related to the damping performance. Generally, in SSD technique using piezoelectric element, the operative energy corresponding to the amplitude of piezoelectric voltage $V$ plays an important role. It corresponds to the available energy to perform the control. Increased available operative energy or increased voltage results in better damping performance.

\subsection{Energy transfer conception}

The active control demonstrates high damping performance, but it usually requires a powerful external energy source to drive the control system. In some practical industrial applications, it is difficult to achieve supply of external energy to the system. Another method is the passive control. It consists of piezoelectric elements connected to an electrical network, which could degrade the mechanical energy of the structure. It does not require any operative energy for damping, but the damping performance would be much lower than active control. To increase the damping performance without external operative energy, several semi-active vibration control techniques have been proposed and developed. The SSD techniques are part of these methods. By using a very small amount of external energy to change its state, a simple switch can modify the dynamic property of a structure by changing the state of an embedded piezoelectric element, thus achieving stiffness change or damping. Only small amounts of energy are necessary to supply the electronic board used for monitoring the vibration and driving the switches.

\section{Non-linear network configuration}

It is seen that whatever the technique considered (SSD techniques or active control techniques); the damping performance is very dependent on the available operative energy related to the piezoelectric elements. This reactive energy, which corresponds to the electrostatic energy stored on the blocked capacitance, quantifies the intensity of the controlling action of the piezoelectric element. In the case of a structure with unwanted modes which are very weakly coupled to the controlling piezoelectric element, the voltage 
or energy requirement could be very high resulting in difficult control of these modes. This energy can be supplied with external sources, but more rationally is extracted from the structure itself in the case of semi-active switched technique. The optimization of this energy could result from a global management of the vibration energy of the structure.

The objective of this work is to propose a novel global approach based on Modal-SSDI and SSDI-Max for energy management and redistribution. In the case of an extended structure with many embedded piezoelectric elements, the proposed strategy consists of using a network implementing a low consumption energy transfer technique relying mainly on switches, diodes and passive elements. The operative energy is extracted from the structure vibration in a given area, on a give vibration mode, and converted into electrostatic energy by so-called source piezoelectric elements. Then this energy is transferred to another piezoelectric element considered as the target one in order to increase its overall charge, all this in a continuous and organized manner. The benefit of the energy transfer network is strongly related to the energy level of the source elements or modes, and more globally to the energy distribution in the smart structure. The objective is to show how the energy in the structure can be managed and redistributed, and to consider the suitable network arrangement.

\subsection{SST and SST-Max configuration}

The piezoelectric elements are arranged in two parts, the three source piezoelectric elements (PZ1, PZ2 and PZ3) and the target piezoelectric element PZ4 targeting by the vibration of the fourth mode of the structure. Each source piezoelectric element transfer its available energy using the left hand side switches. The switches are arranged in pair. One is for the positive voltage; the other is for the negative voltage. Each switch is triggered on the voltage extremum (or eventually on the source modal coordinate) to perform energy transfer toward CTP or CTN (Fig. 3).

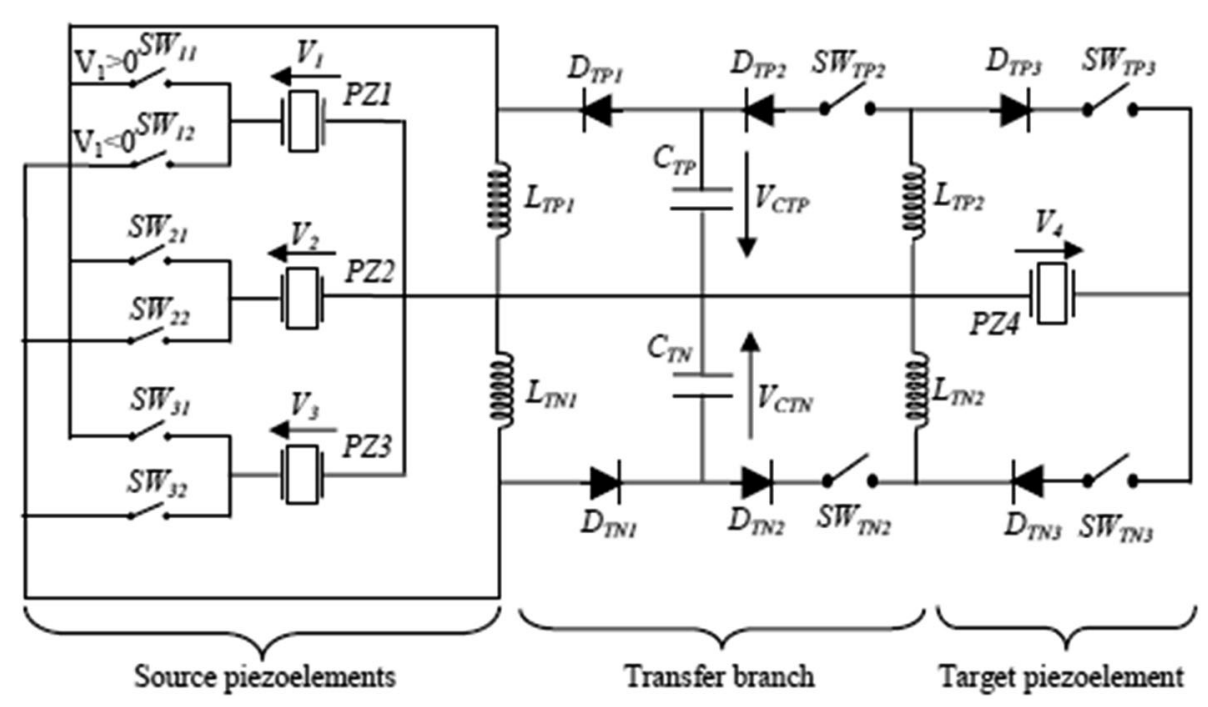

Fig. 3 The extended SST network reassembles the four piezoelectric elements of the smart structure 
The piezoelectric element PZ4, located on the right hand side of the schematic, is the target element of the energy transfer. The inversion of its voltage is triggered following the Modal-SSDI strategy (SST) and the Modal-SSDI-Max strategy (SST-Max) targeted on the $q 4$ modal coordinate. The energy transfer from the storage capacitances is controlled in the same manner as in the basic circuit previously described.

\section{Simulations results}

The simulations are performed using the MATLAB/Simulink software environment.

\subsection{Multimodal sinusoidal excitation}

Figure 4a gives illustration of the benefit of proposed SST-Max network. It shows the operative energy, which is the electrostatic energy stored on the blocked capacitance of the target piezoelectric element PZ4. The considered excitation is a composite multimodal sinusoidal excitation in the centre of the plate. This energy in both case of SST. It appears that following the proposed approach, the peak energy for extended SST-Max is nearly twice, while the mean level is globally improved during the entire transient regime leading consequently to a better damping of the targeted mode displacement $q 4$, as shown on Fig. $4 b$ ). It is interesting to note that different energy harvesting strategies can be implemented: switching on the source piezoelectric element side can be simply triggered on the voltage extreme (SECE harvesting), but the energy can be pumped voluntarily on specific modes of the structure by using the modal coordinates of the structure motion to trigger the harvesting switches. Finally, at it will be show further, the ability of the network for energy transfer can be quantified by the power flow that is established. This power flow is more convenient to quantify the performance of the network for steady state operation when the structure is excited by a $50 \mu \mathrm{s}$ long, $5 \mathrm{~mJ}$ square force pulse.
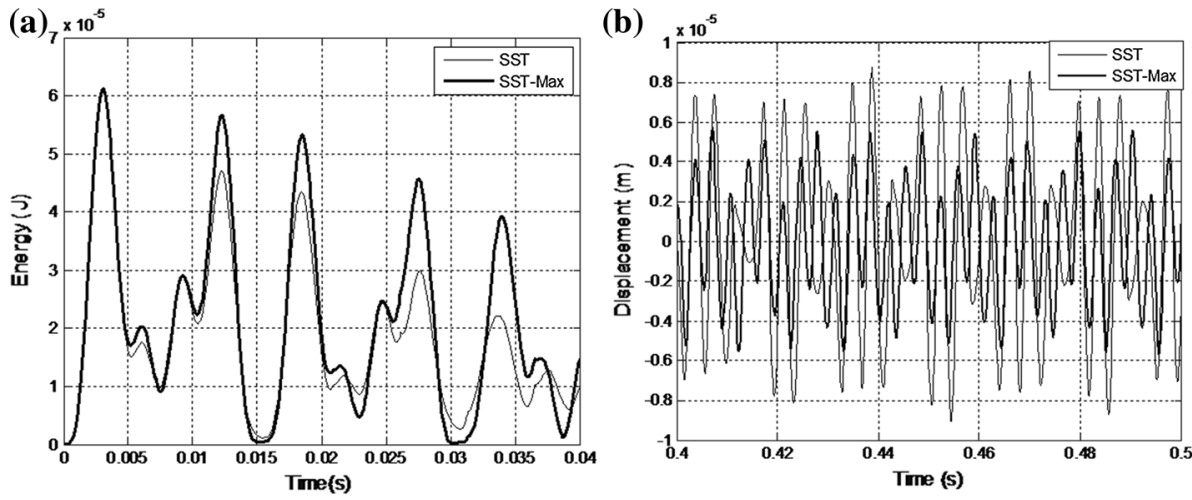

Fig. 4 a The operative energy or electrostatic energy stored on the target piezoelectric element PZ4 blocked capacitance in the case SST and SST-Max configurations of multimodal sinusoidal excitation and b corresponding $q 4$ modal coordinate damping in the case of multimodal sinusoidal excitation 
Fig. 5 Modal damping with a pulse excitation
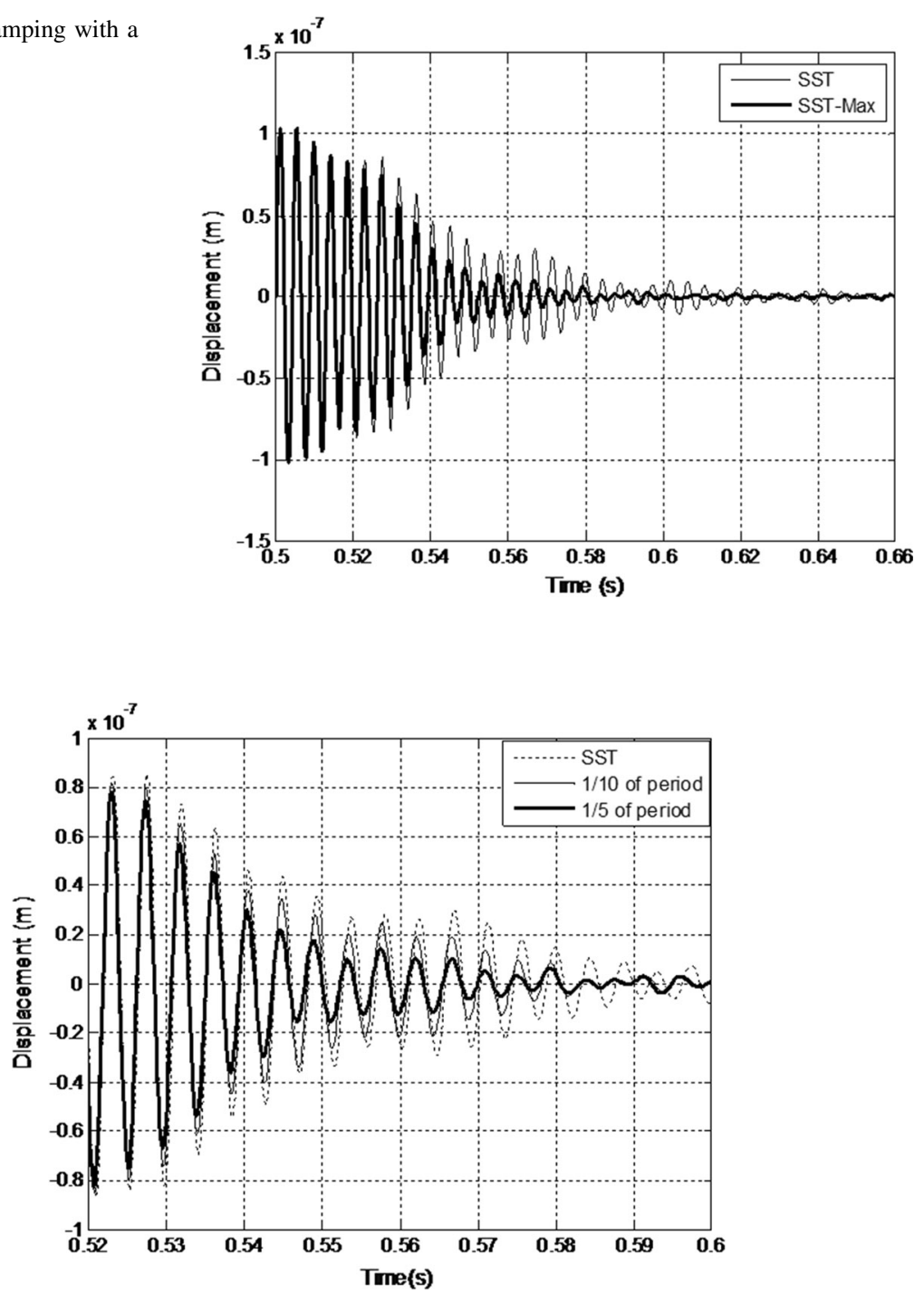

Fig. 6 Influence of the time window for pulse excitation in the time domain

\subsection{Pulse excitation}

The excitation is a wide frequency square force pulse, $50 \mu$ s long and corresponding to $5 \mathrm{~mJ}$ input energy, applied in the centre of the plate structure (Fig. 5).

\subsection{Influence of the time window on the performance of SST-Max}

The time window, which is used to limit the possible time shift prior to switching, is a very important and critic parameter. If it is too small the voltage will not have the possibility to increase, and no significant enhancement will be observed. If it is too long, there is a risk of 
desynchronization of the actuator voltage with the targeted modal speed, thus resulting altered damping. In order to define an optimal time window (Fig. 6).

\section{Conclusion}

In this paper, a novel synchronized switch damping by energy transfer (SST) network of piezoelectric elements is proposed for transferring the energy between source piezoelectric elements and target element. The objective of this work is to propose and evaluate the performance of a network of piezoelectric elements for improved semi-active damping of a smart structure. The basic idea is that semi-active damping is an interesting technique, but it relies on a good electromechanical coupling of the piezoelectric actuator. If the coupling is weak, it is possible to increase the damping by supplying this energy externally. Now the vibrating structure can be used as a source of energy if it is equipped with a network of piezoelectric elements and a low power energy redistribution technique allowing the transfer of operative energy toward the chosen piezoelectric actuator. SST and SST-Max techniques are proposed and will be compared on this basis using a clamped plate equipped with four piezoelectric elements as a benchmark. Modal SST-Max simulations results showed neatly improved damping performances compared to SST for the control of a single mode of the structure, in the case of sinusoidal excitation on the four modes of the plate, pulse, or sinus excitation. Remarkable gains in attenuation lying between 5 and $10 \mathrm{~dB}$ were obtained. Finally, the influence of the maximum time delay between the targeted modal coordinate extremum and the switch instant was evaluated, and the results emphasize the importance of the energy of the targeted mode compared to the other modes of the structure on the definition of the optimal time window.

\section{References}

Badel, A., et al.: Piezoelectric vibration control by synchronized switching on adaptive voltage sources: towards wideband semi-active damping. Acoust. Soc. Am. 119, 2815-2825 (2006)

Chérif, A., Richard, C., Guyomar, D., Belkhiat, S., Meddad, M.: Simulation of multimodal vibration damping of a plate structure using a modal SSDI-Max technique. J. Intell. Mater. Syst. Struct. 23(6), 675-689 (2012)

Chérif, A., Richard, C., Guyomar, D., Belkhiat, S., Meddad, M., Eddiai, A., Hajjaji, A.: Optimization of the piezoelectric transformer by the non-linear methods. Opt. Quantum Electron. (2013). doi:10.1007/ s11082-013-9712-2

Chérif, A., Richard, C., Guyomar, D., Meddad, M., Eddiai, A., Boughaleb, Y., Migalska-Zalas, A., Zawadzka, A., Hajjaji, A., Sahraoui, B.: Multimodal vibration damping of a smart beam structure using Modal SSDI-Max technique. Nonlinear Opt. Quantum Opt. Concepts Modern Opt. 47(1-3), 15-32 (2015)

Eddiai, A., Meddad, M., Touhtouh, S., Hajjaji, A., Boughaleb, Y., Guyomar, D., Belkhiat, S., Sahraoui, B.: Mechanical characterization of an electrictive polymer for actuation and energy harvesting. J. Appl. Phys. 111(12), 124115-1-124115-5 (2012)

Guyomar, D., Richard, T., Richard, C.: Sound wave transmission reduction through a plate using piezoelectric synchronized switch damping technique. J. Intell. Mater. Syst. Struct. 19, 791-803 (2008)

Harari, S., Richard, C., Gaudiller, L.: Hybrid active/semi-active modal control of smart structures. In: Proceedings of SPIE SSM, 7288 (2009a)

Harari, S., Richard, C., Gaudiller, L.: Multimodal control of smart structures based on semi-passive techniques and modal observer. Motion Vib. Control 1, 113-122 (2009b)

Ji, H., Qiu, J., Nie, H., Cheng, L.: Semi-active vibration control of an aircraft panel using synchronized switch damping method. Int. J. Appl. Electromagn. Mech. 46, 879-893 (2014). doi:10.3233/JAE-140096

Lefeuvre, E., Badel, A., Petit, L., Richard, C., Guyomar, D.: Semi-passive piezoelectric structural damping by synchronized switching on voltage sources. J. Intell. Mater. Syst. Struct. 17, 653-660 (2006) 
Richard, C., Guyomar, D., Audigier, D., Ching, G.: Semi-passive damping using continuous switching of apiezoelectric device. Proc. SSM Damp. Isol. 3672, 104-111 (1999)

Wu, D., Guyomar, D., Richard, C.: A new global approach using a network of piezoelectric elements and energy redistribution for enhanced vibration damping of smart structure. In: Proceedings SPIE Conference, Active and Passive Smart Structures and Integrated Systems, vol. 8688, pp. 1-15 (2013) 